[7] W. Krassowska and M. S. Kumar, "The role of spatial interactions in creating the dispersion of transmembrane potential by premature electric shocks," Ann. Biomed. Eng., vol. 25, pp. 949-963, 1997.

[8] J. B. White, G. P. Walcott, A. E. Pollard, and R. E. Ideker, "Myocardial discontinuities: A substrate for producing virtual electrodes that directly excite the myocardium by shocks," Circulation, vol. 97, pp. 1738-1745, 1998.

[9] J. Wall, N. A. Trayanova, K. Skouibine, and W. Krassowska, "Modeling induction of reentry with a realistic S2 stimulus," IEEE EMBS, p. 154, 1999.

[10] D. W. Frazier, W. Krassowska, P.-S. Chen, P. D. Wolf, N. D. Danieley, W. M. Smith, and R. E. Ideker, "Transmural activations and stimulus potentials in three-dimensional anisotropic canine myocardium," Circ. Res., vol. 63, pp. 135-146, 1988.

[11] C. S. Henriquez, "Simulating the electrical behavior of cardiac tissue using the bidomain model," Crit. Rev. Biomed. Eng., vol. 21, pp. 1-77, 1993.

[12] B. J. Roth, W.-Q. Guo, and J. P. Wikswo, Jr., "The effects of spiral anisotropy on the electric potential and the magnetic field at the apex of the heart," Math. Biosci., vol. 88, pp. 191-221, 1988.

[13] B. J. Roth," "Mechanism for polarization of cardiac tissue at a sealed boundary," Med. Biol. Eng. Comput., vol. 37, pp. 523-525, 1999.

[14] P.-S. Chen, P. D. Wolf, E. G. Dixon, N. D. Danieley, D. W. Frazier, W. M. Smith, and R. E. Ideker, "Mechanism of ventricular vulnerability to single premature stimuli in open-chest dogs," Circ. Res., vol. 62, pp. 1191-1209, 1988.

[15] D. W. Frazier, W. Krassowska, P.-S. Chen, P. D. Wolf, E. G. Dixon, W M. Smith, and R. E. Ideker, "Extracellular field required for excitation in three-dimensional anisotropic canine myocardium," Circ. Res., vol. 63, pp. 147-164, 1988

[16] P.-S. Chen, P. D. Wolf, S. D. Melnick, N. D. Danieley, W. M. Smith, and R. E. Ideker, "Comparison of activation during ventricular fibrillation and following unsuccessful defibrillation shocks in open-chest dogs," Circ. Res., vol. 66, pp. 1544-1560, 1990.

[17] R. J. Sweeney, R. M. Gill, M. I. Steinberg, and P. R. Reid, "Ventricular refractory period extension caused by defibrillation shocks," Circulation, vol. 82, pp. 965-972, 1990.

[18] A. S. L. Tang, P. D. Wolf, F. J. Claydon, III, W. M. Smith, T. C. Pilkington, and R. E. Ideker, "Measurement of defibrillation shock potential distributions and activation sequences of the heart in three dimensions," Proc. IEEE, vol. 76, pp. 1176-1186, Sept. 1988.

[19] S. B. Knisley, N. Trayanova, and F. Aguel, "Roles of electric field and fiber structure in cardiac electric stimulation," Biophys. J., vol. 77, pp. 1404-1417, 1999.

[20] B. J. Roth, "Mechanisms for electrical stimulation of excitable tissue," Crit. Rev. Biomed. Eng., vol. 22, pp. 253-305, 1994.

[21] _ _ "Electrical conductivity values used with the bidomain model of cardiac tissue," IEEE Trans. Biomed. Eng., vol. 44, pp. 326-328, Apr. 1997.

[22] S. J. Worley, W. M. Smith, and R. E. Ideker, "Construction of a multipolar electrode system referenced and anchored to endocardium for study of arrhythmias," Amer. J. Physiol., vol. 250, pp. H530-H536, 1986.

[23] S. B. Knisley, W. M. Smith, and R. E. Ideker, "Effect of field stimulation on cellular repolarization in rabbit myocardium: Implications for reentry induction," Circ. Res., vol. 70, pp. 707-715, 1992.

[24] N. Shibata, P.-S. Chen, E. G. Dixon, P. D. Wolf, N. D. Danieley, W M. Smith, and R. E. Ideker, "Influence of shock strength and timing on induction of ventricular arrhythmias in dogs," Amer. J. Physiol., vol. 255, pp. H891-H901, 1988.

[25] J. M. Davidenko, P. F. Kent, D. R. Chialvo, D. C. Michaels, and J. Jalife, "Sustained vortex-like waves in normal isolated ventricular muscle," Proc. Nat. Acad. Sci., vol. 87, pp. 8785-8789, 1990.

[26] P.-S. Chen, Y.-M. Cha, B. B. Peters, and L. S. Chen, "Effects of myocardial fiber orientation on the electrical induction of ventricular fibrillation,” Amer. J. Physiol., vol. 264, pp. H1760-H1773, 1993.

\section{A Statistical Technique for Measuring Synchronism Between Cortical Regions in the EEG During Rhythmic Stimulation}

Antonio Mauricio F. L. Miranda de Sá, Antonio Fernando C. Infantosi*, and David M. Simpson

Abstract - The coherence function has been widely applied in quantifying the degree of synchronism between electroencephalogram (EEG) signals obtained from different brain regions. However, when applied to investigating synchronization resulting from rhythmic stimulation, misleading results can arise from the high correlation of background EEG activity. We, thus propose a modified measure, which emphasizes the synchronized stimulus responses and reduces the influence of the spontaneous EEG activity. Critical values for this estimator are derived and tested in Monte Carlo simulations. The effectiveness of the method is illustrated on data recorded from 12 young normal subjects during rhythmic photic stimulation.

Index Terms-Coherence, EEG, rhythmic stimulation, synchrony measure.

\section{INTRODUCTION}

Rhythmic photic stimulation (RPS) is used as a diagnostic tool in clinical neurophysiology, since abnormal patterns in the electroencephalogram (EEG) may be emphasized during RPS [1]. Its use in conjunction with coherence estimates has been proposed by a number of authors [2]-[5], since the stimuli may reduce the variability of mental states [5], and show distinct patterns of groups whose background EEG at rest is similar [2]-[4]. However, in quantifying synchronization of EEG signals due to RPS (or other rhythmic stimuli) the strong coherence of background EEG from neighboring areas is a confounding influence. This coherence results from activation of wide cortical areas and spreading of the electrical fields through the conducting layers between cortex and scalp [6]. Thus, high coherence estimates at the frequency of stimulation do not necessarily indicate a synchronized response to stimulation, but could largely reflect correlation of the spontaneous activity [7]. An alternative measure of synchronization, less dependent on background activity is, therefore, proposed. This could provide a measure of cortical connectivity (as does coherence), but one that is specific to the stimulus responses. The method is based on coherence between stimuli and EEG responses, which has been proposed and used by Dobie and Wilson [8], [9] in the detection of auditory evoked potentials at individual EEG derivations and, recently, was also applied in somatosensory stimulation [10]. In Section II, this new approach is developed, together with a test of significance for synchronization between two cortical regions due to the stimuli. These are then evaluated in Monte Carlo simulations, from which confidence limits can also be obtained. The application of the methods on EEG data collected from 12 young normal subjects during RPS illustrates the advantages of the new technique over the conventional approach.

Manuscript received October 5, 2000; revised June 10, 2001. This work was supported by the CNPq and PRONEX/Ministry of Science and Technology, Brazil. Asterisk indicates corresponding author

A. M. F. L. Miranda de Sá is with the Department of Electricity, Federal Institution of Higher Education of São João del Rei, São João del Rei, MG, 36300-000, Brazil.

*A. F. C. Infantosi is with the Biomedical Engineering Program, Federal University of Rio de Janeiro, P.O. Box 68510, 21945-970, Rio de Janeiro, RJ, Brazil (e-mail: afci@peb.ufrj.br).

D. M. Simpson is with the Department of Medical Physics, Leicester Royal Infirmary, Leicester LE1 5WW, U.K.

Publisher Item Identifier S 0018-9294(01)08288-X. 


\section{The ESTIMATOR OF SYNCHRONIZATION $\hat{\kappa}_{1,2}^{2}(f)$}

The conventional coherence between discrete-time signals $x[k]$ and $y[k]$ is given in terms of their Fourier transforms as

$$
\gamma_{x y}^{2}(f)=\frac{\left|\lim _{T \rightarrow \infty} \frac{1}{T} E\left[X^{*}(f) Y(f)\right]\right|^{2}}{\lim _{T \rightarrow \infty} \frac{1}{T} E\left[|X(f)|^{2}\right] \cdot \lim _{T \rightarrow \infty} \frac{1}{T} E\left[|Y(f)|^{2}\right]}
$$

where $X(f)$ and $Y(f)$ are $T$-length Fourier transforms of the signals; "*" indicates the complex conjugate; and $E[\bullet]$ is the expectation operator.Its estimate, using the familiar approach of dividing the signals into $M$ segments, is given as

$$
\hat{\gamma}_{x y}^{2}(f)=\frac{\left|\sum_{i=1}^{M} X_{i}^{*}(f) Y_{i}(f)\right|^{2}}{\sum_{i=1}^{M}\left|X_{i}(f)\right|^{2} \cdot \sum_{i=1}^{M}\left|Y_{i}(f)\right|^{2}}
$$

where "^" denotes an estimate; $X_{i}(f)$ and $Y_{i}(f)$ are the $T$-length Fourier transforms of the $i$ th windowed data segments; and $M$ is the number of segments used in the estimation. When $x[k]$ is identical (deterministic) in each data-window $X_{i}(f)$ can be removed from the sum in (2), leading to the modified estimator

$$
\hat{\kappa}_{y}^{2}(f)=\frac{\left|\sum_{i=1}^{M} Y_{i}(f)\right|^{2}}{M \sum_{i=1}^{M}\left|Y_{i}(f)\right|^{2}} .
$$

As $M \rightarrow \infty$, this becomes

$$
\kappa_{y}^{2}(f, T)=\frac{|E[Y(f, T)]|^{2}}{E\left[|Y(f, T)|^{2}\right]} .
$$

Whereas in (1) $T \rightarrow \infty$, it may be noted that here $T$ is fixed. The estimate in (3) has been used under the name of magnitude squared coherence (MSC) in the detection of the response to auditory and somatosensory stimuli [8]-[10]. Similar to coherence, the $\hat{\kappa}_{y}^{2}(f)$ approaches zero in the absence of synchronization between the stimuli and EEG signals. With increasing strength of responses, $\hat{\kappa}_{y}^{2}(f)$ increases and when $Y_{i}(f)$ is constant for all windows, i.e., when $y[k]$ contains only stimulus responses without additional noise, $\hat{\kappa}_{y}^{2}(f)=1$. It can be shown, using the results of Akaike [11] and Brillinger [12, p. 190], and with some further manipulation, that

$$
(M-1) \frac{\hat{\kappa}_{y}^{2}(f)}{1-\hat{\kappa}_{y}^{2}(f)} \sim F_{2,2(M-1)}^{\prime}(\lambda)
$$

where " " denotes "is distributed as" and $F_{2,2(M-1)}^{\prime}(\lambda)$ is the noncentral F-distribution [13, p. 29] with 2 and $2(M-1)$ degrees of freedom (DOFs), and noncentrality parameter $\lambda$ given as

$$
\lambda=2 M \frac{\kappa_{y}^{2}(f)}{1-\kappa_{y}^{2}(f)} .
$$

For the null-hypothesis of no response the right-hand side of (5) reduces to the (central) F distribution with 2 and $2(M-1)$ DOF, since $\kappa_{y}^{2}(f)=$ 0 for this particular case. This allows significance levels and critical values for $\hat{\kappa}_{y}^{2}(f)$ to be calculated, in accordance with [10].

$\hat{\kappa}_{y}^{2}(f)$ should not, strictly, be considered an estimate of coherence when both signals contain the same periodic activity, as at the frequency of oscillation coherence always assumes a value of one and its use is,

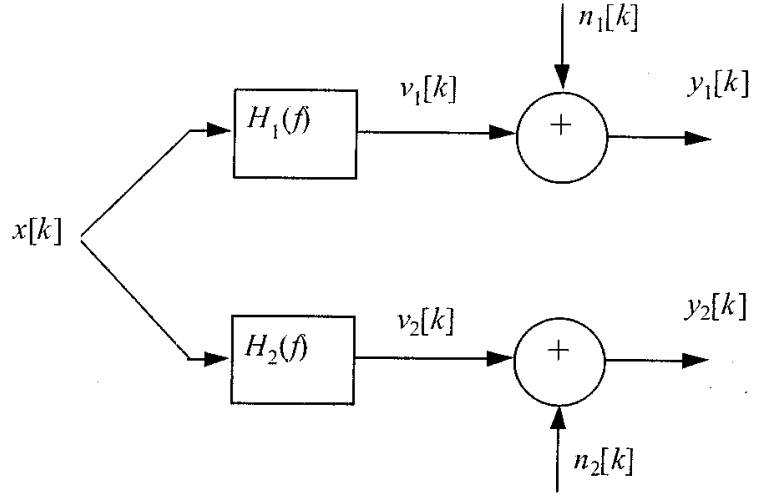

Fig. 1. Linear model used in deriving $\hat{\kappa}_{1,2}^{2}(f) . x[k]$ is the stimulus, $v_{1}[k]$ and $v_{2}[k]$ are the stimulus responses [output of the filters $H_{1}(f)$ and $\left.H_{2}(f)\right]$, and $n_{1}[k]$ and $n_{2}[k]$ are the contributions of background activity to the measured EEG signals $y_{1}[k]$ and $y_{2}[k]$.

therefore, inappropriate. It can however readily be shown that $\kappa_{y}^{2}(f)$ reflects the signal-to-noise ratio (SNR) of the response in the frequency bin centered on $f$ (and width defined by the window-length $T$ ) and, thus, provides a useful tool in quantifying the strength of stimulusresponses.

In addition to this use in individual EEG derivations, $\hat{\kappa}_{y}^{2}(f)$ can also form the basis for an alternative measure of synchronization between different cortical regions during stimulation, as shall now be shown. Based on Lopes da Silva [14] and in common with much work in this area, we will assume throughout the following analysis that spontaneous background EEG signals are stationary (for the epoch lengths of interest) and Gaussian random signals.

In the model of Fig. 1, coherence between $y_{1}[k]$ and $y_{2}[k]$ is given by $[15$, p. $167-168]$

$$
\gamma_{y_{1} y_{2}}^{2}(f)=\gamma_{x y_{1}}^{2}(f) \cdot \gamma_{x y_{2}}^{2}(f)
$$

assuming $n_{1}[k], n_{2}[k]$, and $x[k]$ are uncorrelated. Now let $x[k]$ represent the pulse-train of periodic stimuli, which are filtered in $H_{1}(f)$ and $H_{2}(f)$ to give the (steady-state) evoked potentials at the two EEG derivations $y_{1}[k]$ and $y_{2}[k]$. The "noise" inputs $n_{1}[k]$ and $n_{2}[k]$ represent the background EEG activity, which are considered Gaussian, stationary, additive and independent of the stimulus responses. A measure of coherence in the stimulus responses of $y_{1}[k]$ and $y_{2}[k]$ may then be defined similarly to (7)

$$
\hat{\kappa}_{1,2}^{2}(f)=\hat{\kappa}_{y_{1}}^{2}(f) \cdot \hat{\kappa}_{y_{2}}^{2}(f) .
$$

This is proposed as a means of quantifying cortical connectivity in stimulus responses, and can be used in a similar manner to coherence as applied to background EEG activity. Values approaching unity indicate that the cerebral regions under study are closely coupled. As with $\hat{\kappa}_{y}^{2}(f), \hat{\kappa}_{1,2}^{2}(f)$ is strongly dependent on the window-length and should not strictly be considered a coherence estimate, but values obtained with the same windows-lengths $T$ from different subjects, stimuli, cortical regions or frequencies can be compared. Since both $\hat{\kappa}_{y 1}^{2}(f)$ and $\hat{\kappa}_{y 2}^{2}(f)$ reflect the strength (SNR) of the deterministic stimulus responses $\left(v_{1}[k]\right.$ and $v_{2}[k]$ in Fig. 1$), \hat{\kappa}_{1,2}^{2}(f)$ is relatively immune to the strength of coherence of background EEG activity. In the investigation of stimulus responses, it is this reduced dependence of $\hat{\kappa}_{1,2}^{2}(f)$ on the coherence of background EEG activity $\left(n_{1}[k]\right.$ and $\left.n_{2}[k]\right)$ that is the major advantage of this measure over conventional coherence.

Critical values for $\hat{\kappa}_{1,2}^{2}(f)$ can be obtained for the null hypothesis $\mathrm{H}_{0}: H_{1}(f)=H_{2}(f)=0$, i.e., the absence of stimulus responses is $y_{1}[k]$ and $y_{2}[k]$. Following an approach similar to that used in deriving (5) and with an appropriate change of variables, it was shown in [16] 
that the probability density function (PDF) of $\left[1-\hat{\kappa}_{1,2}^{2}(f)\right] / \hat{\kappa}_{1,2}^{2}(f)$ is given as

$$
\begin{aligned}
& p\left(\frac{1-\hat{\kappa}_{1,2}^{2}}{\hat{\kappa}_{1,2}^{2}}\right)=\int_{0}^{\left(1-\hat{\kappa}_{1,2}^{2}\right) / \hat{\kappa}_{1,2}^{2}} \\
& \frac{p_{F}\left(\frac{\frac{1-\hat{\kappa}_{1,2}^{2}}{\hat{\kappa}_{1,2}^{2}}-W_{2}}{(M-1)\left(W_{2}+1\right)}\right) \cdot p_{F}\left(\frac{W_{2}}{M-1}\right)}{(M-1)^{2}\left|W_{2}+1\right|} d W_{2}
\end{aligned}
$$

where $p_{F}(\Omega)$ is the PDF of an F-distribution with $2(M-1)$ and 2 DOFs. From (9), critical values or the statistical significance of a given estimate $\hat{\kappa}_{1,2}^{2}(f)$ can be determined, under the null-hypothesis of no stimulus response.

In the above, it was assumed that $n_{1}[k]$ and $n_{2}[k]$ are independent. Perfect coherence between these signals is the alternative extreme case and leads to the following simpler expression [16]

$$
p\left(\frac{1-\hat{\kappa}_{1,2}^{2}(f)}{\hat{\kappa}_{1,2}^{2}(f)}\right)=\frac{p_{F}\left(\frac{-1+\sqrt{1+\left[1-\hat{\kappa}_{1,2}^{2}(f)\right] / \hat{\kappa}_{1,2}^{2}(f)}}{M-1}\right)}{2(M-1) \sqrt{1+\left[1-\hat{\kappa}_{1,2}^{2}(f)\right] / \hat{\kappa}_{1,2}^{2}(f)}}
$$

where $p_{F}(\Omega)$ is defined as in (9).

For intermediate values of background EEG correlation, the sampling distribution and critical values for $\hat{\kappa}_{1,2}^{2}(f)$ become more difficult to evaluate. In the following simulation study, this case will be investigated, and results compared to those of the extremes given by (9) and (10).

\section{Simulation STUdies}

\section{A. Critical Values}

Monte Carlo simulations based on the model of Fig. 1 with $\mathrm{H}_{0}$ : $H_{1}(f)=H_{2}(f)=0$ were carried out, taking $y_{1}[k]=n_{1}[k]$ and $y_{2}[k]=n_{2}[k] . \hat{\kappa}_{1,2}^{2}(f)$ was calculated from 10000 pairs of $5 \leq M \leq$ 40 independent signals. Fig. 2 shows the ninety-fifth percentiles of the resultant sample distribution, which are virtually identical to those derived from (9). The equivalent was then carried out for $y_{1}[k]=y_{2}[k]=$ $n_{1}[k]$, and compared with the critical values obtained from (10), again showing excellent agreement. The percentiles for correlation values $\left(r^{2}\right.$ between $n_{1}[k]$ and $\left.n_{2}[k]\right)$ of 0.5 and 0.75 are also displayed in Fig. 2, and as expected lie at values intermediate to those given by (9) and (10). It may be noted that the critical values for $r^{2}=1$ may be taken as the "worst case," such that if any $\hat{\kappa}_{1,2}^{2}(f)$ exceeds this critical value, the estimate is significant at least at the level $\alpha$.

\section{B. Confidence Limits}

Next, estimates of confidence limits for $\hat{\kappa}_{1,2}^{2}(f)$ were obtained by Monte Carlo methods. For the case of $H_{1}(f)=H_{2}(f)$ and equal power in $n_{1}[k]$ and $n_{2}[k]$, sets of $\hat{\kappa}_{y, j}^{2}(f)$ were simulated from independent random variables $\left(\varphi_{j}, j=1,2\right)$ that follow a noncentral-F-distribution. Based on $(5), \hat{\kappa}_{y}^{2}, j(f)$ was obtained from (11)

$$
\hat{\kappa}_{y, j}^{2}(f)=\frac{\varphi_{j}}{M-1+\varphi_{j}} \quad j=1,2 .
$$

The noncentrality parameter $\lambda$ for $\varphi_{j}$ was chosen, according to (6), with $M=5,10,30$, and 60 and for $\kappa_{y, j}^{2}(f)$ of $0, \sqrt{0.1}, \sqrt{0.2}$,

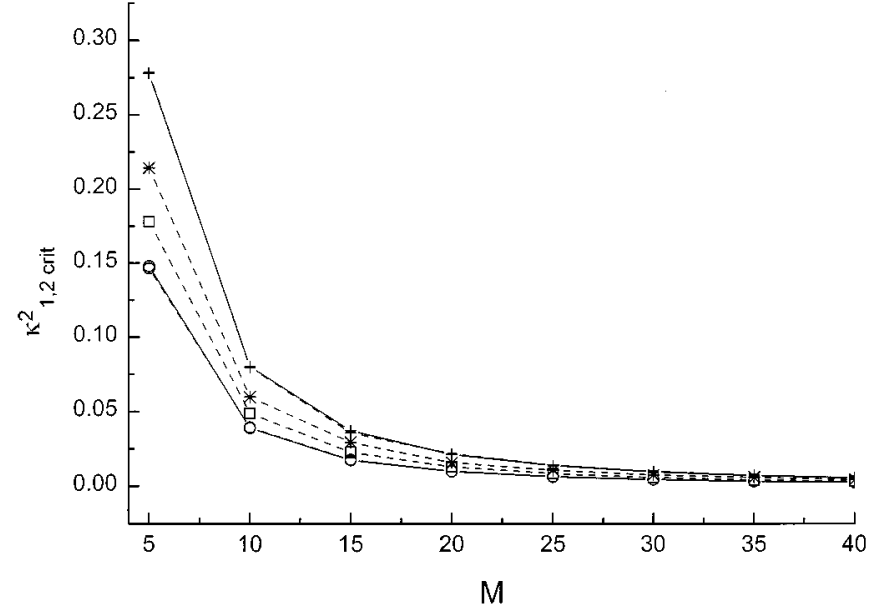

Fig. 2. Critical values $(\alpha=5 \%)$ for $\hat{\kappa}_{1,2}^{2}(f)$ obtained from theory (continuous lines) and results of simulations (dashed lines) for different correlation $r^{2}$ between $n_{1}[k]$ and $n_{2}[k]: r^{2}=1$ (“+”) $r^{2}=0.75$ (“*”), $r^{2}=$ 0.5 (“ $\square$ "), and $r^{2}=0$ (“o").
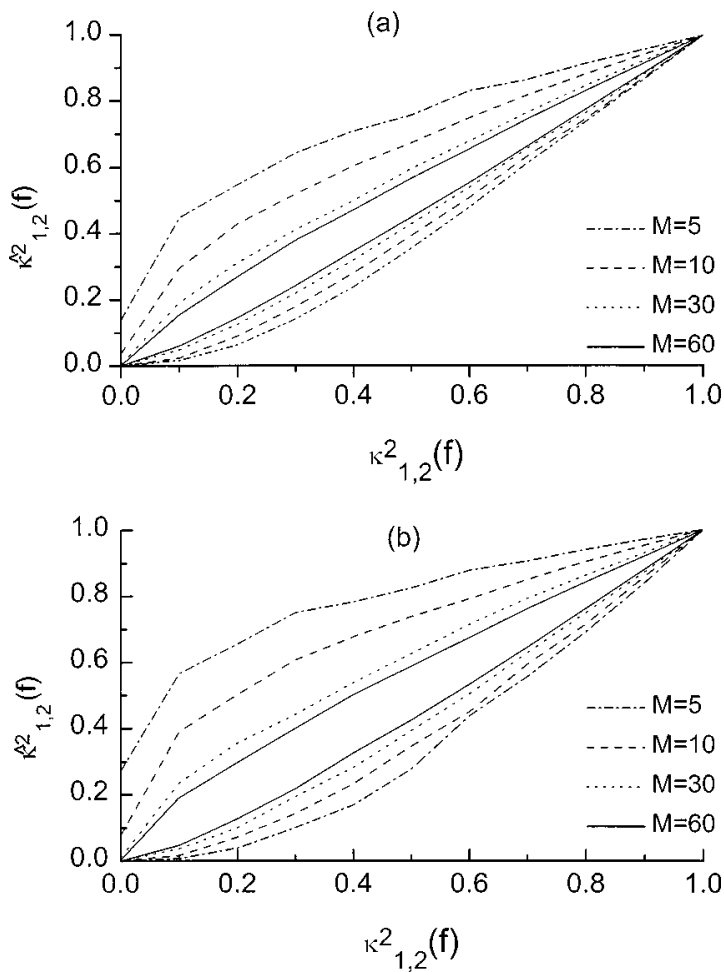

Fig. 3. Confidence limits for $\hat{\kappa}_{1,2}^{2}(f)$-(a) uncorrelated background activity, (b) identical background activity.

$\sqrt{0.3}, \ldots, 1$. Pairs of independent values of simulated $\hat{\kappa}_{y, j}^{2}(f)$ were then multiplied to form $\hat{\kappa}_{1,2}^{2}(f)=\hat{\kappa}_{y_{1}}^{2}(f) \cdot \hat{\kappa}_{y_{2}}^{2}(f)$. The $95 \%$ confidence limits were then obtained as the 2.5th and the 97.5th percentiles of the distribution of estimates from an ensemble of 10000 values of $\hat{\kappa}_{1,2}^{2}(f)$. These limits correspond to the case of uncorrelated noise inputs, $n_{1}[k]$ and $n_{2}[k]$ in the model of Fig. 1 , as the $\hat{\kappa}_{y, j}^{2}(f)$ were generated from independent samples.

The case of perfect identical background activities and $H_{1}(f)=$ $H_{2}(f)$ was then considered by taking $\varphi_{1}=\varphi_{2}$ in (11). Confidence limits for $\hat{\kappa}_{1,2}^{2}(f)$ were then obtained in the same way as above, but now $\hat{\kappa}_{1,2}^{2}(f)=\left[\hat{\kappa}_{y_{1}}^{2}(f)\right]^{2}$.

Fig. 3 shows the confidence limits for $\hat{\kappa}_{1,2}^{2}(f)$ for uncorrelated [Fig. 3(a)] and perfectly correlated background activities [Fig. 3(b)]. 
(a)
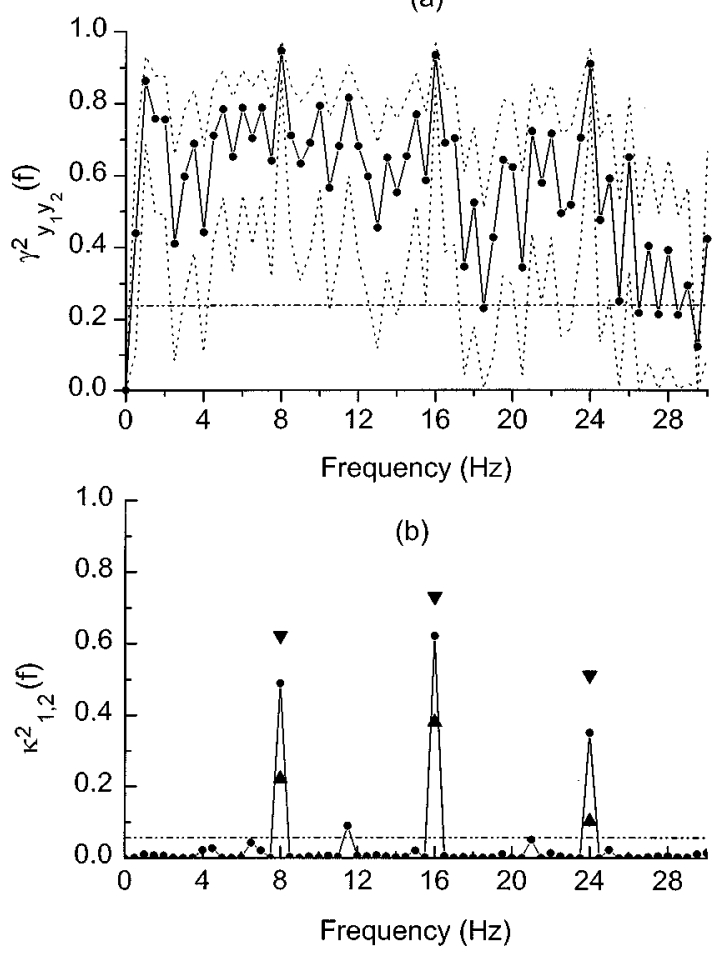

Fig. 4. (a) Coherence $\hat{\gamma}_{u_{1}}^{2}(f)$ and (b) $\hat{\kappa}_{1,2}^{2}(f)$ for one subject during photic stimulation at $8 \mathrm{~Hz}(M \stackrel{12}{=})$. The horizontal dash-dotted lines indicate the critical values. Confidence limits in (a) are shown as dotted lines and in (b) by triangles (harmonics of stimulation only). Critical values in (b) were obtained from the simulations of Fig. 3(b).

As may be expected, the first case in which the $\hat{\kappa}_{y, j}^{2}(f)$ are independent (and, therefore, have higher DOFs), leads to narrower confidence bands.

\section{APPLICATION TO EEG DURING RPS}

In order to demonstrate the performance of $\hat{\kappa}_{1,2}^{2}(f)$ and compare it to that of $\hat{\gamma}_{y_{1} y_{2}}^{2}(f)$, the EEG signal of 12 normal young subjects (age range: 9-17 years) was recorded over a period of $24 \mathrm{~s}$ during stroboscopic flash stimulation at $8 \mathrm{~Hz}$. The signals at the derivations $\mathrm{O} 1$ and $\mathrm{O} 2$ (reference: ipsilateral earlobe) were recorded and digitized at $256 \mathrm{~Hz}$. The coherencies were then calculated following (2) and (8), using $M=12$ epochs each of 2-s duration and applying a rectangular window.

Fig. 4 shows a typical result from one subject together with the confidence limits for $\hat{\gamma}_{y_{1} y_{2}}^{2}(f)$ obtained according to Otnes and Enochson [17, p. 377-378] and for $\hat{\kappa}_{1,2}^{2}(f)$ using the simulation results of the previous section. In Fig. 4(b), confidence limits are only shown at the harmonics of stimulation, and were taken from the Monte Carlo results of Fig. 3(b), which are wider than those of Fig. 3(a). As expected, $\hat{\gamma}_{y_{1} y_{2}}^{2}(f)$ is much higher than $\hat{\kappa}_{1,2}^{2}(f)$, but the latter shows much clearer peaks at the stimulus frequency and its harmonics. The former also shows statistically significant coherence at almost all frequencies displayed, whereas for the latter, significant synchronization as expected is only evident at the harmonics of stimulation and (by a small margin) one other frequency.

The number of subjects in the present sample for whom the interhemispheric coherence $\hat{\gamma}_{y_{1} y_{2}}^{2}(f)$ reached a statistically significant level is depicted in Fig. 5, together with the equivalent results for $\hat{\kappa}_{1,2}^{2}(f)$. Again, the latter gives very different results at the harmonics of stimulation compared to the intermediate frequencies. Both here,

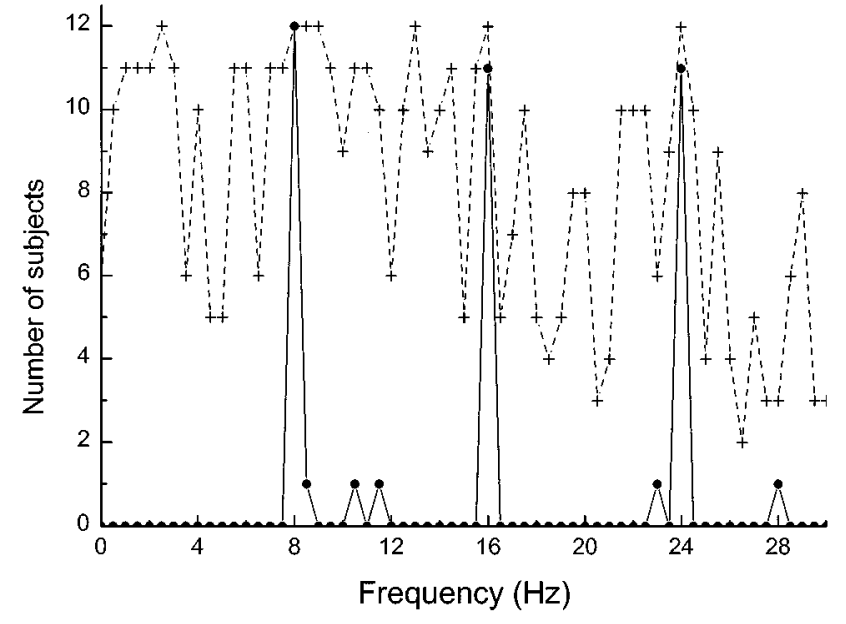

Fig. 5. Histogram for the number of subjects (out of a total of 12) in whom $\hat{\kappa}_{1,2}^{2}(f)$ (continuous line-“"”) and $\hat{\gamma}_{y_{1} y_{2}}^{2}(f)$ (dashed line "+") was significant $(\alpha=5 \%)$.

and in Fig. 4(b), the critical values for $\hat{\kappa}_{1,2}^{2}(f)$ used are those of the worst case scenario of correlated noise.

\section{DISCUSSION AND CONCLUSION}

In this paper, a novel measure of synchronization of the EEG during periodic stimulation has been presented. Its application to EEG signals clearly shows its relative immunity to coherence in background EEG activity, emphasizing the response at the stimulus frequency and its harmonics. It, therefore, provides a powerful tool in the study of cortical connectivity specific to the sensory system under investigation. While $\hat{\gamma}_{y_{1} y_{2}}^{2}(f)$ may be taken as indicating the "strength" of connections between the two cortical sites during background EEG activity, $\hat{\kappa}_{1,2}^{2}(f)$ obtained from the EEG during photic stimulation is more restrictive to structures pertaining to the visual system. Results for $\hat{\gamma}_{y_{1} y_{2}}^{2}(f)$ in the latter application would be strongly biased by background EEG coherence.

The sample distribution, derived under the null hypothesis of no stimulus responses, permits the statistical significance of $\hat{\kappa}_{1,2}^{2}(f)$ to be tested. As expected, the critical values are much lower than those of conventional coherence. For large $M$ these are similar for both perfectly correlated and uncorrelated background EEG $\left(n_{1}[k]\right.$ and $\left.n_{2}[k]\right)$. Since the true correlation of background activity may be unknown, the significance obtained from assuming perfect correlation provides the most conservative (worst case) estimate. Confidence limits cannot readily be derived for $\hat{\kappa}_{1,2}^{2}(f)$ and only simulation results have been shown in the present work. These limits are wider for the case when stimulus-response and background EEG are identical in the two EEG derivations, than for independent noise of equal power. However, confidence limits may change again if $\kappa_{y 1}^{2}(f)$ and $\kappa_{y 2}^{2}(f)$ are allowed to be different, which is the most realistic scenario in practice.

The technique presented was designed for the study of cerebral connectivity under periodic stimulation. The periodicity of the stimuli and responses is however not strictly required. The method may also be used in the case of irregular stimuli, provided that the signal epochs analyzed are synchronized with the trigger and contain only one stimulus/response pair. This is evident from the definition of $\hat{\kappa}_{y}^{2}(f)$ in (3), where $X_{i}(f)$ as used in (2) must be constant for all windows. This is usually achieved with periodic stimuli and regularly spaced windows, but could also be obtained when data windows are aligned with the start of nonuniformly spaced stimuli. This permits the application in such diverse conditions as self-triggered psychophysiological experimental protocols, or the analysis of high frequency ECG signals, where 
the QRS complexes may be taken as the "trigger.” The technique, thus, provides an additional resource with wide potential applicability in the analysis of biomedical signal analysis.

\section{ACKNOWLEDGMENT}

The authors gratefully acknowledge the useful comments by anonymous reviewers of an earlier version of the paper and the assistance from Dr. V. Lazarev and the Laboratory of Clinical Neurophysiology of the Instituto Fernandes Figueira, Rio de Janeiro, in data collection.

\section{REFERENCES}

[1] T. Takahashi, "Activation methods," in Electroencephalography-Basic Principles, Clinical Applications, and Related Fields, 4th ed, E. Niedermeyer and F. Lopes da Silva, Eds. Baltimore, MD: Williams \& Wilkins, 1998, pp. 261-284.

[2] Y. Wada, Y. Nanbu, Y. Kicuchi, Y. Koshino, and T. Hashimoto, “Aberrant functional organization in schizophrenia: Analysis of EEG coherence during rest and photic stimulation in drug-naïve patients," $\mathrm{Neu}$ ropsychology, vol. 38, pp. 63-69, 1998.

[3] Y. Wada, Y. Nanbu, Y. Koshino, N. Yamaguchi, and T. Hashimoto, "Reduced interhemispheric EEG coherence in Alzheimer disease: Analysis during rest and photic stimulation," Alzheimer Dis. Assoc. Disord., vol. 12, no. 3, pp. 175-181, Mar. 1998.

[4] Y. Jin, C. A. Sandman, J. C. Wu, J. Bernat, and S. G. Potkin, "Topographic analysis of EEG photic driving in normal and schizophrenic subjects," Clin. Electroencephal., vol. 26, no. 2, pp. 102-107, Apr. 1995.

[5] J. Kaiser and J. H. Gruzelier, "Timing of puberty and EEG coherence during photic stimulation," Int. J. Psychophysiol., vol. 21, pp. 135-149, 1996.
[6] R. Srinivasan, P. L. Nunes, and R. B. Silberstein, "Spatial filtering and neocortical dynamics: Estimates of EEG coherence," IEEE Trans. Biomed. Eng., vol. 45, pp. 814-826, July 1998.

[7] A. M. F. L. Miranda de Sá, D. M. Simpson, and A. F. C. Infantosi, "A Comparison between source derivation and partial coherence," in Medical and Biological Engineering and Computing: Abstracts World Congress Medical Physics and Biomedical Engineering, vol. 35, Suppl. Part 1,1997 , p. 522.

[8] R. A. Dobie and M. J. Wilson, "Analysis of auditory evoked potentials by magnitude-squared coherence," Ear Hearing, vol. 10, no. 1, pp. 2-13, Feb. 1989.

[9] — - "A comparison of $t$ test, $F$ test, and coherence methods of detecting steady-state auditory-evoked potentials, distortion-product otoacoustic emissions, or other sinusoids," J. Acoust. Soc. Amer, vol. 100, no. 4, pp. 12 236-12246, Oct. 1996.

[10] D. M. Simpson, C. J. Tierra-Criollo, R. T. Leite, J. B. Zayen, and A. F. C. Infantosi, "Objective response detection in an electroencephalogram during somatosensory stimulation," Ann. Biomed. Eng., vol. 28, no. 6 , pp. 691-698, 2000.

[11] H. Akaike, "On the statistical estimation of the frequency response function of a system having multiple input," Ann. Inst. Statist. Math., vol. 17, pp. 185-210, 1965.

[12] D. R. Brillinger, Time Series Analysis and Theory. New York: Holt, Rinehart and Winston, 1975.

[13] S. M. Kay, Fundamentals of Statistical Signal Processing Volume II Detection Theory. Englewood Cliffs, NJ: Prentice-Hall, 1998.

[14] F. Lopes da Silva, "EEG analysis: Theory and practice," in Electroencephalography-Basic Principles, Clinical Applications, and Related Fields, 4th ed, E. Niedermeyer and F. Lopes da Silva, Eds. Baltimore, MD: Williams \& Wilkins, 1998, pp. 1135-1163.

[15] J. Bendat and A. G. Piersol, Engineering Applications of Correlation and Spectral Analysis. New York: Wiley, 1993.

[16] A. M. F. L. Miranda de Sá, "Desenvolvimento de Técnicas para o Estudo da Coerência no EEG durante Foto-Estimulação Intermitente," D.Sc. dissertation, Biomed. Eng. Program - COPPE, Federal Univ. Rio de Janeiro, Rio de Janeiro, Brazil.

[17] R. K. Otnes and L. Enochson, Applied Time Series Analysis, Volume 1: Basic Techniques. New York: Wiley, 1978. 